\title{
Structure and reactivity of Ruthenium nanoparticles
}

\section{Gavnholt, Jeppe; Schiøtz, Jakob}

\section{Published in:}

Physical Review B Condensed Matter

Link to article, DOI:

10.1103/PhysRevB.77.035404

Publication date:

2008

Document Version

Publisher's PDF, also known as Version of record

Link back to DTU Orbit

\section{Citation (APA):}

Gavnholt, J., \& Schiøtz, J. (2008). Structure and reactivity of Ruthenium nanoparticles. Physical Review $B$ Condensed Matter, 77(3), 035404. https://doi.org/10.1103/PhysRevB.77.035404

\section{General rights}

Copyright and moral rights for the publications made accessible in the public portal are retained by the authors and/or other copyright owners and it is a condition of accessing publications that users recognise and abide by the legal requirements associated with these rights.

- Users may download and print one copy of any publication from the public portal for the purpose of private study or research.

- You may not further distribute the material or use it for any profit-making activity or commercial gain

- You may freely distribute the URL identifying the publication in the public portal

If you believe that this document breaches copyright please contact us providing details, and we will remove access to the work immediately and investigate your claim 


\title{
Structure and reactivity of ruthenium nanoparticles
}

\author{
Jeppe Gavnholt and Jakob Schiøtz* \\ Center for Individual Nanoparticle Functionality (CINF), Department of Physics, Technical University of Denmark, \\ DK-2800 Kongens Lyngby, Denmark
}

(Received 6 July 2007; published 3 January 2008)

\begin{abstract}
We present a method for obtaining detailed structural information of ruthenium nanoparticles in at least the diameter range from 1.5 to $5 \mathrm{~nm}$. The method is based on an ensemble approach where a large number of low-energy structures are collected in an ensemble, from which average properties can be extracted using Boltzmann averaging. The method is used to obtain the number of catalytic active step sites present on the surface of the ruthenium particles. We find that the presence of highly catalytic active step sites does not depend significantly on the temperature within a relevant temperature range; the presence of step sites is mainly a function of the lowest energy shape of the cluster, i.e., a function of the number of atoms. By combining the structural information with estimations of the single site activities in the ammonia synthesis, we find that the optimal particle diameter is approximately $3 \mathrm{~nm}$. The single site activities are estimated by using density functional theory to calculate the barrier of the rate limiting step, the dissociation of a nitrogen molecule.
\end{abstract}

DOI: 10.1103/PhysRevB.77.035404

PACS number(s): 61.46.Df, 82.33.Hk, 82.20.Wt

\section{INTRODUCTION}

Within the past decade, it has become possible to make reasonably accurate theoretical estimations of adsorption energies of individual molecules and barrier heights for the different part reactions in a variety of different catalytic processes. ${ }^{1-14}$ This success has been made possible by the use of density functional theory and the increase in computer power. The adsorption energy and barrier height information can be combined with either a mean-field kinetic model ${ }^{11}$ or a kinetic Monte Carlo model, ${ }^{12}$ where interactions between surface species can be included, to obtain the catalytic activity of single sites. The development of these methods has given much insight to the fundamental processes governing many catalytic reactions. One of the things that has been realized is that some catalytic reactions, such as the ammonia synthesis, is completely dominated by a few highly active sites. Dahl et al. ${ }^{13}$ show that the sticking coefficient of a nitrogen molecule on a ruthenium surface drops at least 9 orders of magnitudes, when the steps are blocked by $\mathrm{Au}$ atoms. This is supported by density functional theory calculations, which show that the barrier of the $\mathrm{N}_{2}$ dissociation is much lower at a step site than at a flat surface site. This proves that the detailed structure of the catalyst is of vital importance for at least some catalytic reactions. It is then necessary not only to find the single site activity in order to make theoretical estimates of a catalyst's activity but also to make some estimate of the catalyst's detailed surface structure.

Honkala et al. ${ }^{14}$ made such an estimate of the catalytic activity of ruthenium clusters in the ammonia synthesis and found beautiful agreement with experiments, i.e., between a factor 3 and 20 in difference. However, the structure estimation was only applicable for a few cluster sizes and did not include temperature effects. In this paper we take the step further and develop a method, which can systematically treat all cluster sizes between at least 1.5 and $5 \mathrm{~nm}$ in diameter, and which gives the temperature dependence on the struc- ture. We hope that such a model will provide further insight to the factors governing the presence of highly active sites. In this paper, we have focused on ruthenium clusters. However, the model can be directly applied to other hep metals, and with minor modifications, it could be applied to other crystal structures as well. We have chosen to study ruthenium since this is known to be one of the best catalysts for the ammonia synthesis.

The main principle of the method is for a given cluster size to collect a large ensemble of possible and relevant configurations for the cluster. Average properties of the cluster can then be calculated using simple Boltzmann averaging over the ensemble. In order to do that, we need the energy and the property we want to average of each cluster in the ensemble. In this paper, we have focused on calculating the average number of catalytic active sites; however, in principle, the ensemble could be used to calculate other properties as well. In the following two sections, we describe the method. First, we describe the model for obtaining the energy, and second, we go through the configuration generation and the Boltzmann averaging. In this part, we will also discuss the limitations and approximations of the method. Finally, we present the results, where we look at the number of catalytic active sites as a function of cluster size. Here, we will also carry out calculations on the barrier size for the dissociation of nitrogen at different sites in order to link the obtained structures to actual catalytic activities for the ammonia synthesis. The dissociation of nitrogen is the rate limiting step in the ammonia synthesis, i.e., knowledge of the barrier height for this dissociation will enable us to make a simple approximation of the ammonia synthesis rate.

\section{ENERGY OF A CLUSTER}

Both in the process of finding the relevant cluster configurations and in the following Boltzmann averaging, it is of absolute importance that we are able to calculate the energy of the different cluster configurations. Since this paper fo- 
cuses on large clusters with up to 4000 atoms in size, it is not possible to apply high-accuracy methods such as density functional theory directly for this task. We need a method which can compute the energy of a 4000 atom cluster within a few tenths of a second, such that it is possible to obtain energies of many different structures within a reasonable time of computation. One possibility is to optimize an effective medium theory (EMT) potential for ruthenium, as described by Jacobsen et al. ${ }^{15,16}$ Another possibility is to assign an energy to each atom in the cluster depending on the configuration of nearest neighbors, where the total energy is then simply the sum of all these atomic energies. The individual atomic energies should then be estimated from density functional theory (DFT). This turned out to be the preferred method for this type of calculations, so in the following, we will describe this method in more detail. Afterward, we argue why this is the preferable method by making a comparison to the above-mentioned effective medium approach.

All the DFT calculations presented in this paper are based on a plane-wave expansion of the wave functions, a revised Perdew-Burke-Ernzerhof (RPBE) description of exchange and correlation effects, ${ }^{17}$ and ultrasoft pseudopotentials. ${ }^{18}$ Plane waves with energy up to $350 \mathrm{eV}$ are used. The selfconsistent electron density is determined by iterative diagonalization of the Kohn-Sham Hamiltonian, Fermi population of the Kohn-Sham states using a temperature of $0.1 \mathrm{eV}$, and Pulay mixing of the resulting electronic density. ${ }^{19}$ All total energies have been extrapolated to zero temperature. Slabs have been separated by at least $10 \AA$ in all calculations. The DFT calculations are made with the DACAPO software..$^{20}$

\section{A. Description of the method}

The simplest way to assign an energy to each atom is to let the energy depend solely on the number of nearest neighbors. DFT is then used to obtain a relation between the energy and the number of nearest neighbors. However, since different surfaces in many cases contain atoms with the same number of neighbors, we extend this model a bit, such that the atomic energy not only depends on the number of nearest neighbors but also the configuration of these. In this way, we preserve the freedom to assign different energies to different surfaces. However, we also restrict ourselves to find energies of nonstrained hcp structures. As it will be argued in the next section, this is sufficient for our purpose.

We illustrate the method by the example shown in Fig. 1 where we assign energies to the atoms sitting on the (0001) and (10 $\overline{1} 1)$ surfaces and the edge between them. Furthermore, we include the step formation energy. First, the bulk energy is determined by doing a standard lattice parameter optimization using DFT. Here, we found the lattice parameters $a=2.7529 \AA$ and $c=4.3595 \AA$, which are $1.7 \%$ and $1.8 \%$ above the experimental values. The lattice parameter values obtained here have been used in all the following calculations. Furthermore, the found bulk energy has been subtracted from the following results, such that all energies are the total surface energies. After this, the surface energies are determined by calculating the energies of the slabs shown in panels (a) and (b) in Fig. 1. All atoms with 12 nearest

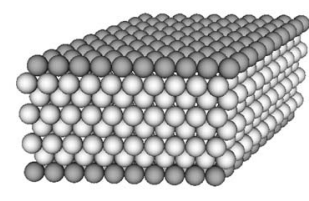

a

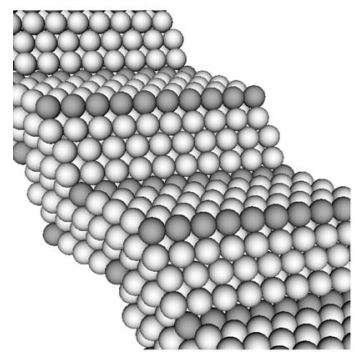

C
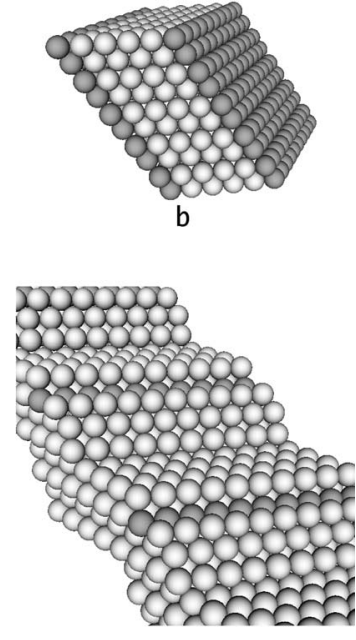

d
FIG. 1. Some of the surfaces for which energies have been calculated using DFT. The calculations are used to determine the energy of the gray atoms. Panel (a) shows the close-packed surface (0001). Panel (b) shows the (10ī1) surface. The surfaces on panels (c) and (d) are repeated edges between the (0001) surface and the

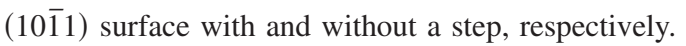

neighbors are considered as bulk atoms, i.e., they are assigned zero energy. All the energies are then placed on the rest of the atoms. For the (0001) surface, this is straightforward since all the remaining atoms sit in the same configuration, so the energy is just distributed evenly. However, for the $(10 \overline{1} 1)$ surface, there are two different types of surface atoms. In this case, we choose to distribute the surface energy proportional to the missing number of nearest neighbors. After this, we find the edge energy by calculating the energy of the slab shown in panel (c) in Fig. 1, which consists of repeated edges between the (0001) surface and the (1011) surface. From the figure, it is clear that two types of atoms have not been assigned an energy, the ones in the inner and the ones in the outer edge. Again, we distribute the remaining energy among these atoms, proportionally to the number of missing nearest neighbors. Finally, we can find the step formation energy by calculating the energy of the slab shown in panel (d) in Fig. 1, where a step has been formed at the edge by removing a row of atoms. Here, there is only one type of atom, which has not been assigned an energy. By assigning the remaining energy to these, we ensure that the model gives correct step formation energies.

We did these calculations for the (1010A), (0001), and (1011) surfaces and all the edges between these since the ruthenium clusters almost entirely consist of these lowenergy surfaces. Furthermore, we included the $(10 \overline{10 B})$ surface. Naturally, there will still be atoms sitting on the clusters in other configurations than those included here. These atoms have been assigned an energy based on the number of nearest neighbors alone. We obtain a relation between the energy and the number of nearest neighbors by averaging the already calculated energies. The atoms which are assigned an energy in this way are mainly corner atoms, i.e., it is a small part of the total energy. 


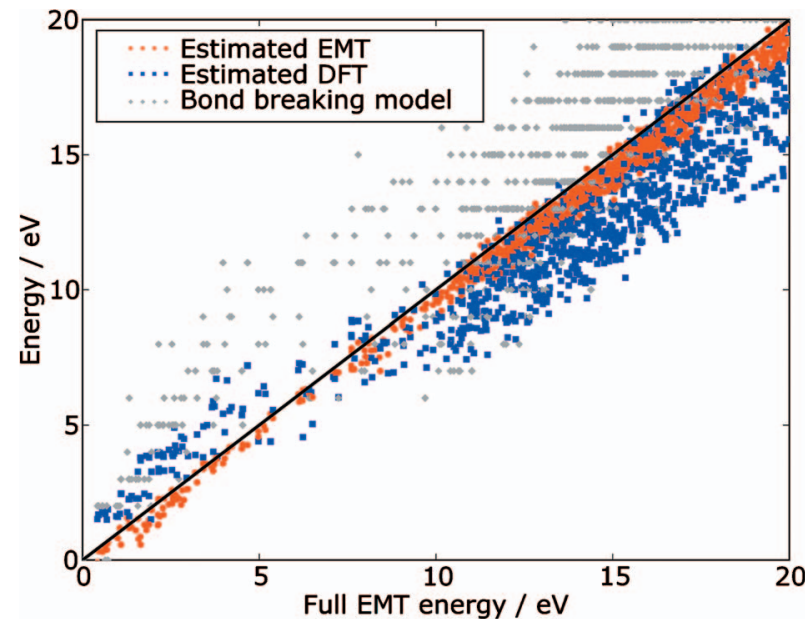

FIG. 2. (Color) A comparison between the energy obtained using four different methods. The estimated EMT, the estimated DFT, and the simple bond breaking model are all compared to the full EMT energy. Each point corresponds to a certain configuration of a 10046 atom cluster. Points on black line indicate perfect agreement between the full EMT energy and the method in question. Energies are relative to the energy of the found ground state configuration. From this, we conclude that the estimated DFT is the preferred method in our situation (see text).

\section{B. Test of the method}

Since it is impossible to calculate the DFT energy of a large cluster, it is hard to test the accuracy of this method directly. So, instead we optimized an EMT potential for ruthenium. $^{21}$ With this potential, we went through all the same calculations as for the DFT potential and established an estimated EMT potential. With these three potentials, the estimated DFT, the estimated EMT, and the full EMT, we calculated the energy of a lot of different configurations of a 10046 atom cluster. In Fig. 2, we have plotted the obtained energies using the estimated DFT and the estimated EMT versus the full EMT energy. For comparison, we also included a simple bond breaking model, where the energy is proportional to the number of broken nearest neighbor bonds. The figure shows that the estimated EMT model and the full EMT potential have better agreement than any of the others. From this, we conclude that the main difference between the full EMT potential and the estimated DFT potential is due to the change from EMT to DFT and not due to using the estimated DFT values instead of the exact values. In other words, calculating the cluster energy as a sum of atomic energies, which are estimated from surface calculations, is a better approximation than calculating the cluster energy using the EMT potential instead of the accurate DFT potential.

Another advantage of the estimated DFT model is that we are sure to get the high-accuracy DFT step formation energies at the edges, which are the most important energies in this case, since these step edge sites typically are the most catalytic active, as we will argue for later. These steps are badly described in the EMT model.

Finally, we tested the estimated DFT potential by calculating the energy of the $(30 \overline{3} 1)$ and $(1 \overline{100})$ surfaces, which
TABLE I. Comparison between full DFT surface energy and the estimated DFT surface energy of two surfaces, which were not used in the development of the estimated DFT potential.

\begin{tabular}{lccc}
\hline \hline Surface & $\begin{array}{c}\text { Full DFT } \\
(\mathrm{eV} / \text { atom })\end{array}$ & $\begin{array}{c}\text { Estimated DFT } \\
(\mathrm{eV} / \text { atom })\end{array}$ & $\begin{array}{c}\text { Error } \\
(\mathrm{eV} / \text { atom })\end{array}$ \\
\hline$(30 \overline{3} 0)$ & 2.525 & 2.536 & 0.011 \\
$(1 \overline{1} 00)$ & 2.377 & 2.371 & -0.006 \\
\hline \hline
\end{tabular}

was not used in estimating the individual atomic energies. The results are shown in Table I, from which it is seen that the error per surface atom is very low, on the order of $0.01 \mathrm{eV} /$ surface atom. All cluster energies in the following are determined using the estimated DFT model.

\section{NUMBER OF SITES ON A CLUSTER}

In this section, we present the developed method for finding the number of times a specific site occurs on a cluster of a given number of atoms. First, we go through the method of obtaining a ensemble of configurations for the cluster, and then we show how this ensemble can be used to obtain the number of sites at a given temperature using simple Boltzmann factor averaging. Finally, we perform some testing of the method.

\section{A. Generating an ensemble}

Clusters of several thousand atoms in size have almost endless different possible configurations, so it is completely impossible to calculate the energy of them all. Instead, we need a method which only searches through relevant configurations, i.e., configurations of low energy. The obvious choice in such a situation is some sort of Monte Carlo method, and we have in fact tried using the Metropolis algorithm. All close-packed configurations were allowed and the energy of each configuration was calculated using the EMT potential. By all close-packed configurations, we mean hcp and fcc including all possible stacking faults. Each new configuration was generated by moving one atom from one part of the cluster to another. The algorithm was able to find configurations with stacking faults, but they were too high in energy to contribute significantly to the ensemble.

The problem using such a method is that the potential energy surface contains many local minima with very high barriers between them, which can make the time it takes to search through a sufficient amount of the configurational space much too high except for the smallest clusters, unless one makes sure it is possible to jump directly through these barriers. Also, an algorithm based on genetic optimization was implemented, but it suffered from the same problems.

The limitations of these methods, however, inspired a way around the problems since they showed that all these lowenergy configurations, in which the simulations got stuck, have some common characteristics. They are all clusters,

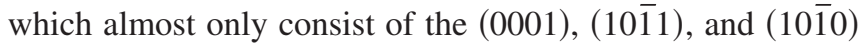
surfaces, possibly with some atoms missing near the edges or 


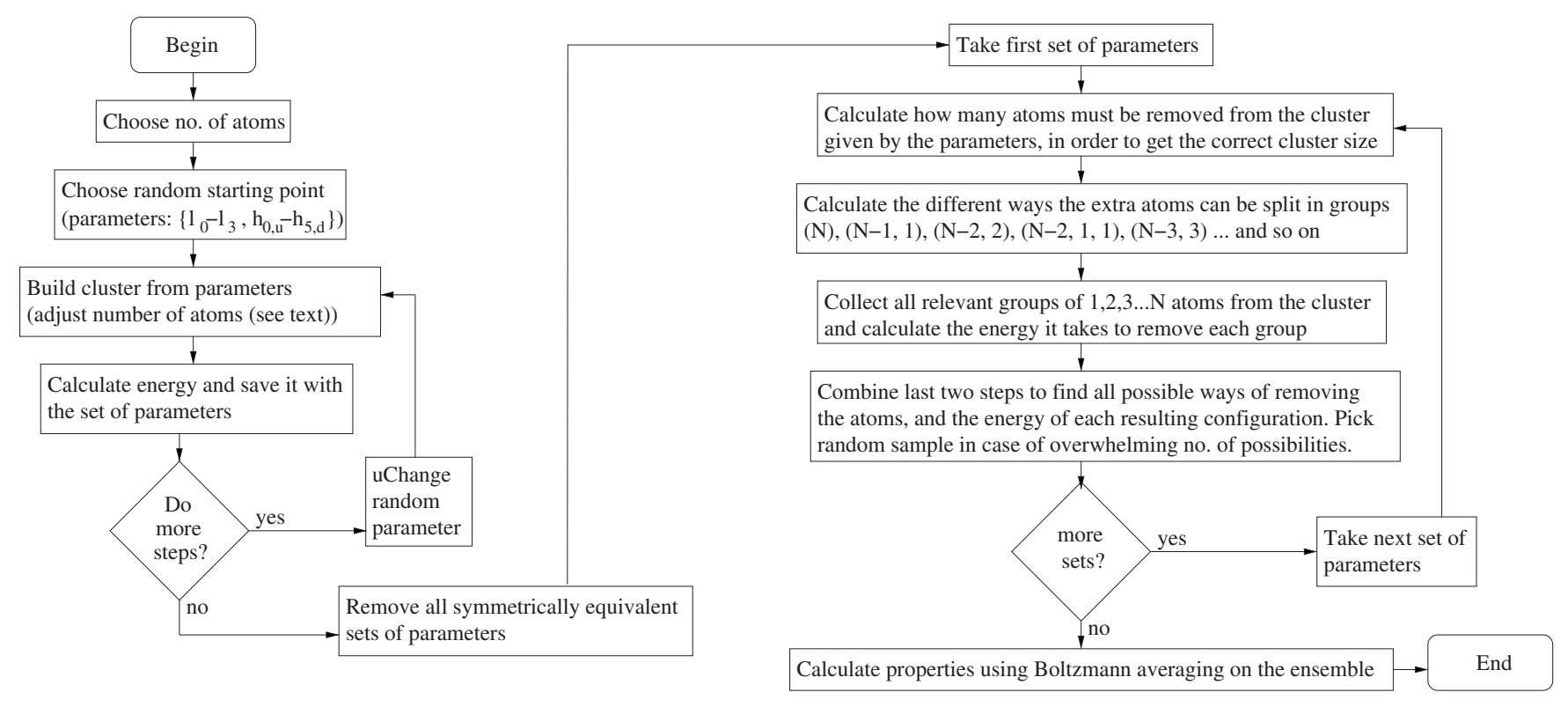

FIG. 3. Flowchart of the algorithm that collects an ensemble of relevant structures for a given cluster.

in a few cases a couple of adatoms placed on one of the surfaces. Because of this, we can restrict ourselves to search through configurations with these characteristics. The reason that only configurations with these characteristics are relevant is that the energy it takes to break a nearest neighbor bond is close to $0.5 \mathrm{eV}$, which is much higher than the thermal energy at all relevant temperatures. So, all odd configurations with holes in the surface or other cuts in the surface are way too high in energy.

We have not investigated the energy of non-close-packed structures, for example, structures with internal stress, which could be relevant for the smallest nanoparticles. However, high-resolution transmission electron microscopy studies of industrial $\mathrm{Ru}$-based catalysts have shown nanoparticles with a regular hcp crystal structure. ${ }^{14,22}$

After having gained these experiences, we chose to divide the generation of the ensemble of structures into two steps. First, we find all the relevant clusters, which only consist of the $(0001),(10 \overline{1} 1)$ and $(10 \overline{1} 0)$ surfaces and with a number of atoms close to the desired cluster size. For each relevant cluster, we then adjust the number of atoms. If the number of atoms is too large, we search through the different ways one can remove the excess number of atoms; otherwise, we search through the ways one can add the lacking number of atoms.

A flowchart of the method is shown in Fig. 3, the details are described in the following sections.

\section{Finding relevant cluster shapes}

The first step is done by describing all relevant clusters through 16 parameters $\left\{l_{0}-l_{3}, h_{0, u}-h_{5, u}, h_{0, d}-h_{5, d}\right\}$, which are defined on Fig. 4. The total height of the cluster is not included since that is governed by the number of atoms. Any

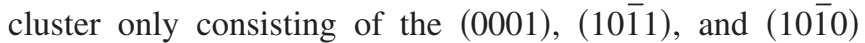
surfaces can be described by these 16 parameters. With this parametrization, it is now possible to escape the large barriers in the potential energy surface, i.e., it is possible to do a Metropolis Monte Carlo search through the configurational space, where one of the parameters is changed between each step. The energies used in the algorithm are not the energies of the cluster given by the 16 parameters since these cluster may not consist of the right number of atoms. Instead, the extra or lacking numbers of atoms have been removed or added at a favorable position on the cluster before calculating the energy.

Through a testing phase, it was found that a simulation temperature of $2 \mathrm{eV}$ is optimal in this type of simulations. At this temperature, the simulations move rather freely between all relevant sets of parameters without spending too much time in irrelevant sets of parameters. The algorithm is run for a sufficient amount of steps, typically around 50 000, and then we pick out the sets of parameters with an energy below $5 \mathrm{eV}$ above the lowest found energy. This limit was set to $5 \mathrm{eV}$ since the removal and/or adding of extra and/or lacking
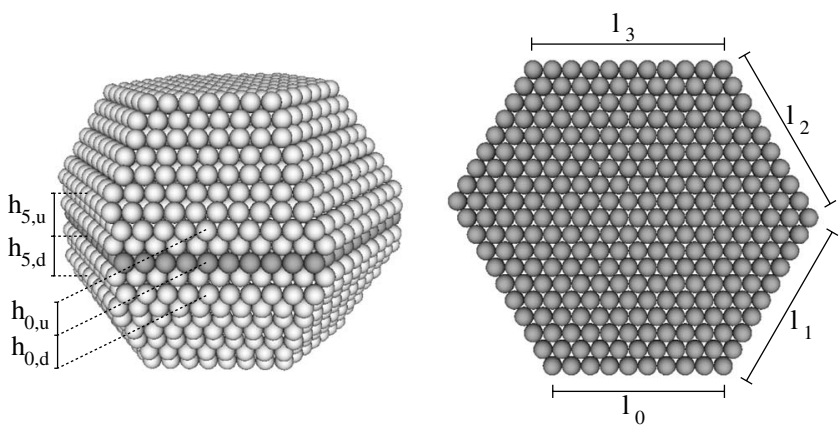

FIG. 4. The definition of the 16 parameters $\left\{l_{0}-l_{3}, h_{0, u}\right.$ $\left.-h_{5, u}, h_{0, d}-h_{5, d}\right\}$ describing the cluster shape. $\left\{l_{0}-l_{3}\right\}$ are the dimensions of the basal plane, whereas $\left\{h_{0, u}-h_{5, u}\right\}$ and $\left\{h_{0, d}-h_{5, d}\right\}$ are the number of layers up and down to the edges between the (0001) surfaces and (10 $\overline{1} 1)$ surfaces. 
atoms may not have been done in the optimal way; i.e., in the next part where we search through all the ways one can do this removal and/or adding, we may find lower energy configurations within these 16 parameters. After some testing, we found that it sometimes is possible to find configurations with up to $2 \mathrm{eV}$ lower energy, i.e., by setting a $5 \mathrm{eV}$ limit, we are reasonably sure to get all configurations with up to $3 \mathrm{eV}$ higher energy than the ground state configurations. Later, we will see that this is enough.

We continue the simulations until we are confident that all relevant sets of parameters have been collected. This we test by looking at how many rotation symmetric and mirror symmetric pairs we have collected. Here, we find that more than half of all the symmetric versions are found, i.e., we feel confident that we rarely miss all the symmetric versions of a relevant configuration. After this, the collected set is reduced, such that it only contain symmetrically different clusters.

\section{Removing excess atoms}

After having collected all relevant sets of parameters, we then go through each set of parameters one by one and figure out how many different configurations each set of parameters correspond to, i.e., typically how many ways we can remove the excess number of atoms. Here, we only go through the ways to remove extra atoms since it is by far the most common situation and since the algorithm is very similar to the one that finds the ways to add lacking atoms.

If the excess number of atoms is $N$, then one could remove all $N$ atoms from one part of the cluster but one could also remove $N-1$ atoms from one part and 1 from another or $N-2$ from one part and 2 from another and so forth. Furthermore, one could remove from three, four, five, and so on different parts of the cluster. In order to include all these possibilities, we start out by collecting all the ways one can remove one atom, a group of two atoms, a group of three atoms, and so on up to a group of $N$ atoms. By a group, we mean atoms sitting next to each other. We only collect groups sitting near the edges, as illustrated in Fig. 5, since all others give too high an energy to be relevant.

After this, we go through all the different ways you can remove groups of atoms such that it adds up to $N$ atoms, i.e., one group of $N$ atoms, two groups of $N-n$ and $n$ atoms, and so forth up to $N$ groups of 1 atom. For each of these different ways, we use the already obtained data on the different ways the different group sizes can be removed and go through all combinations. In some cases, when $N$ is large, the number of combinations is overwhelming. In these cases, we take a random set of combinations out, typically a set of 10000 combinations for each group combination. In these cases, each of the random combinations are assigned a weighting factor of the number of configurations divided by 10000 , which is used in the Boltzmann averaging later such that we get the correct weighing between the configurations when we calculate properties of the clusters. Figure 5 shows a few different configurations for a 1670 atoms cluster. Panel (d) shows the found ground state configuration.

When we have gone through this for all the relevant sets of parameters, we have a huge set of configurations, which is

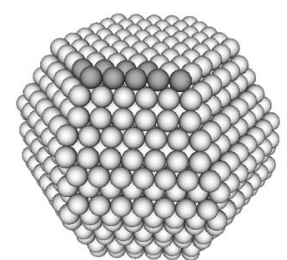

a

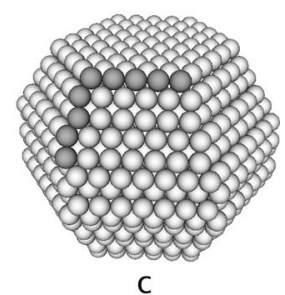

C

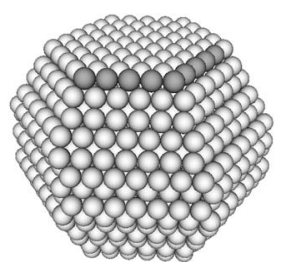

b

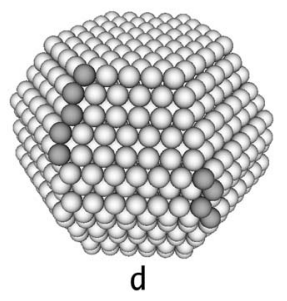

FIG. 5. Some different ways, one could remove nine atoms (gray) from a cluster. (a)-(c) show examples where all removed atoms are in one group. (d) shows an example where the atoms are removed in two groups (one of five and one of four atoms). The configuration shown in (d) is the found ground state configuration for a 1670 atom cluster.

a representative segment of all possible configurations with an energy below a certain limit value.

\section{B. Calculating properties}

After having generated the ensemble of configurations, we can start calculating properties of the cluster. This is done using simple Boltzmann averaging, where the average value of a property $O$ is calculated as

$$
\langle O\rangle=\frac{\sum_{i} O_{i} w_{i} \exp \left[-\frac{E_{i}}{k_{B} T}\right]}{\sum_{i} w_{i} \exp \left[-\frac{E_{i}}{k_{B} T}\right]},
$$

where the sum goes over all configuration in the ensemble. $O_{i}, w_{i}$, and $E_{i}$ are the value of property $O$, the weighting factor, and the energy of configuration number $i$. The weighting factor is the weighting factor introduced in the last section multiplied by a symmetry correction, which is the number of rotations and mirrorings which will result in a new configuration. This is included since the ensemble of configurations has been reduced such that configurations which only differ by a rotation or mirroring are removed. $T$ is the temperature at which we want to know the average. The only property we calculate the average of here is the number of some specific sites. However, we could calculate many other properties of the clusters as well.

This way of averaging the configurations rely on the approximation that it is reasonable to only include configurations which are local minima on the potential energy surface in our ensemble. Ideally, we should also include the different entropies of the different minima. However, we used the developed EMT potential (see Sec. II) and compared a long constant temperature molecular dynamics simulation with 


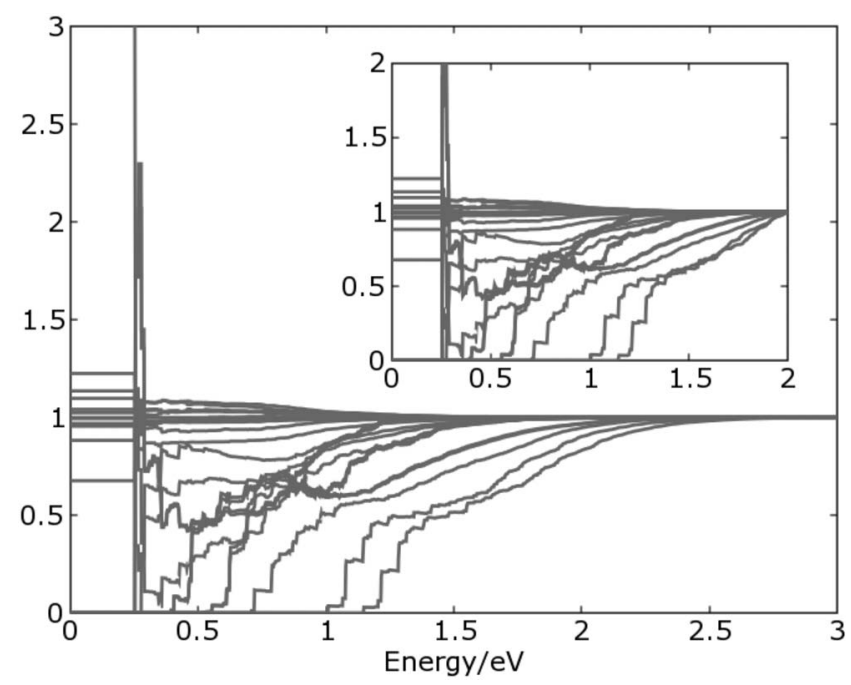

FIG. 6. Only configurations with a energy below a limit value are saved and used in the further calculations. The graph shows the found number of sites divided by the found number of sites with a limit energy of $3 \mathrm{eV}$ above the ground state level for a 4000 atoms cluster as a function of the limit energy. Each line corresponds to a certain site type. The inset shows the same thing where the found number of sites are divided by the found number of sites with a limit energy of $2 \mathrm{eV}$ instead of $3 \mathrm{eV}$. The asymptotic behavior when approaching $3 \mathrm{eV}$ shows that $3 \mathrm{eV}$ is a sufficiently high limit, whereas the inset shows that a limit of $2 \mathrm{eV}$ is insufficient.

the ensemble approach for a small cluster of 79 atoms and found that the difference was below $5 \%$. This indicates that the curvatures of the different local minima are sufficient alike, such that the entropy does not vary much from minimum to minimum, i.e., the approximation seems valid.

\section{Testing the method}

When we collect configurations for the ensemble, we only include configurations which have an energy below a certain limit value. This is done to keep the amount of data down and is justified by the fact that low-energy configurations are more relevant than higher-energy configurations. In order to set this limit value, we made a calculation on a 4000 atom cluster and calculated the number of sites at $1200 \mathrm{~K}$ for 22 different types of sites with many different limit values. We chose this large cluster and this high temperature since we wanted an upper boundary on the limit value. Figure 6 shows the found number of sites relative to the found number of sites at a limit value of $3 \mathrm{eV}$ as a function of the limit value. The graph clearly indicates asymptotic behavior toward 1 as the limit value goes toward $3 \mathrm{eV}$, i.e., we conclude that a limit value of $3 \mathrm{eV}$ above the found ground state energy is sufficient. This is the limit value we have used in all further calculations. The inset in Fig. 6 shows the same plot, but here the number of sites is taken relative to the number of sites at a limit value of $2 \mathrm{eV}$. This graph does not show the same asymptotic behavior, i.e., a limit value of $2 \mathrm{eV}$ is not sufficient.

There are two parts of the method that rely on random sampling. First of all, there is the Monte Carlo part where we

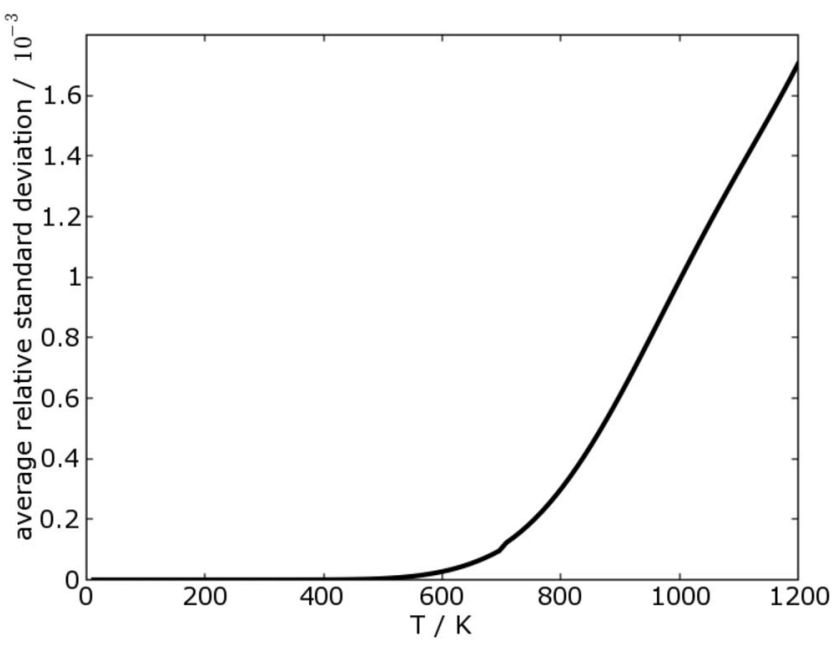

FIG. 7. An estimation of the statistical uncertainty in the calculations. The abundance of 22 different sites has been estimated in three completely independent calculations on a 4000 atoms cluster. For each site, the three estimations on the number of sites have been used to calculate the relative standard deviation. The graph shows the average of these relative standard deviations taken over the 22 different sites as a function of temperature. The low values (below $2 \times 10^{-3}$ show that the statistical error is negligible).

expect we have found all relevant sets of parameters. Furthermore, there is the second part where we rely on random sampling of configurations. In order to check the statistical uncertainty that come from these random samplings, we made three separate simulations on a 4000 atoms cluster. For all three ensembles, we calculated the relative standard deviation on the number of sites for 22 different sites. Figure 7 shows the average of these relative standard deviations as a function of temperature. It is seen that the average relative standard deviation is below $2 \times 10^{-3}$ at all temperatures below $1200 \mathrm{~K}$, i.e., we conclude that the statistical error is negligible.

\section{CALCULATING THE REACTIVITY}

When calculating the catalytic activity of a cluster, one should ideally pick out every type of surface site present on the cluster and calculate the contribution to the catalytic activity from each of these. However, since the catalytic activity is completely dominated by a few highly active sites, we can neglect the contribution from all other sites and only focus on these. In the following, we present the obtained results. First, we show the size dependence of the number of active sites. Second, we combine these results with obtained barrier heights for the dissociation of nitrogen in order to see how the ammonia synthesis activity depends on the cluster size.

\section{A. Number of active sites}

In the dissociation of nitrogen, we expect the dominating sites to be step sites since they possess low-coordinated surface atoms, which from the $d$-band model $^{23,24}$ are predicted 


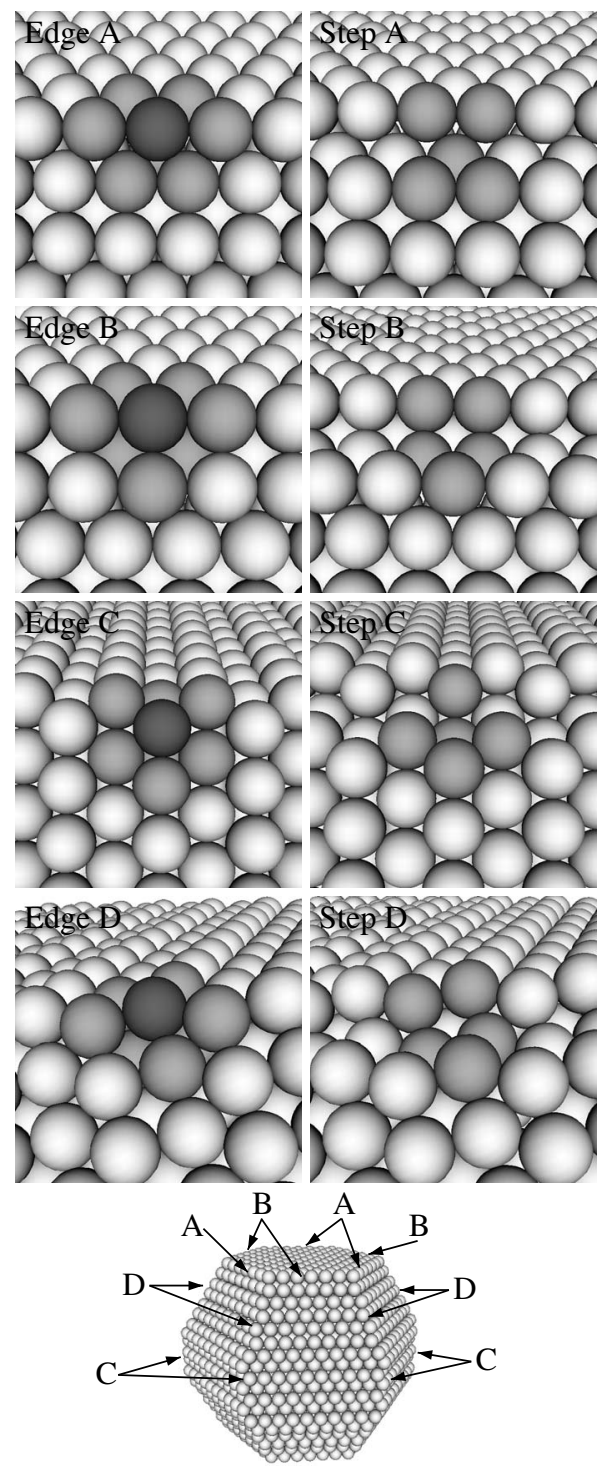

FIG. 8. Definitions of four different edges. Completed edges are seen to the left and stepped edges to the right. The color differences indicate the definitions of the sites. A completed edge site is defined by a dark gray atom having exactly the nearest neighbors sitting as the light gray atoms does. A stepped edge site is defined by a free position having exactly the nearest neighbors sitting as the light gray atoms. Edges A and B connect the (0001) surfaces and (1011) surfaces. Edge C connects two (1010A) surfaces. Edge D connects two $(10 \overline{1} 1)$ surfaces. Step site B is known as the $B_{5}$ site.

to give the largest weakening of the bond. Furthermore, the nitrogen molecule can bind to more surface atoms at the foot of a step. ${ }^{13}$ For this reason, we have focused our analysis in the four step sites shown in Fig. 8. These step sites are most likely to be found at the edges of the cluster, so for comparison we have also shown the same edges without the step in Fig. 8.

Figure 9 shows the average number of these four step sites as a function of the number of atoms in the cluster in the range between 396 and 416 atoms. The black and gray lines correspond to temperatures of 300 and $1200 \mathrm{~K}$, respectively. From the graphs, it is clear that this is a regime where
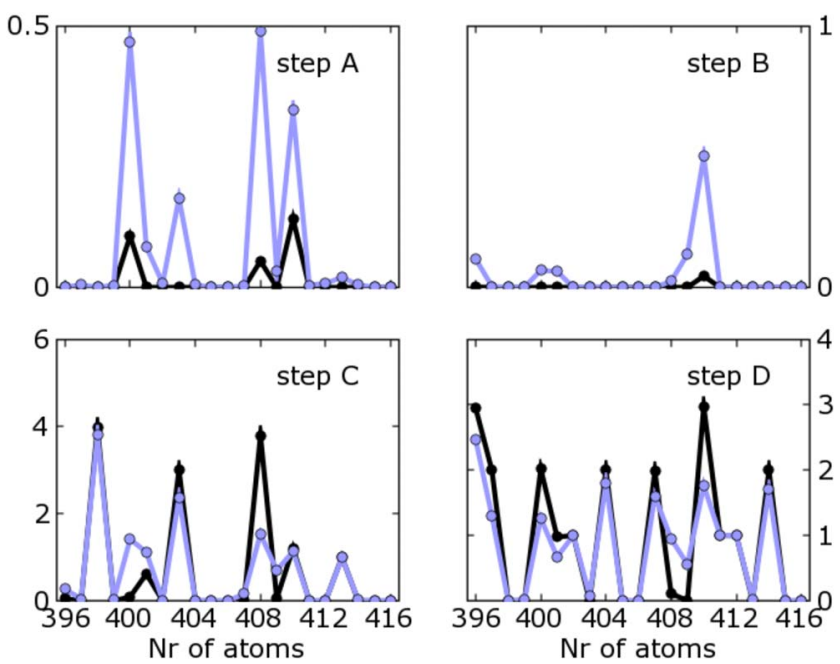

FIG. 9. (Color online) The number of step sites as a function of the number of atoms in the cluster. The four step sites are defined in Fig. 8. The black curve is at a temperature of $300 \mathrm{~K}$, whereas the gray (light blue) is at $1200 \mathrm{~K} .400$ atoms corresponds to a cluster with approximately $1.8 \mathrm{~nm}$ diameter.

the addition or removal of a single atom has a huge impact on the properties of the cluster. This is an expected behavior at the nanometer scale and is one of the many challenges and opportunities that comes when decreasing the size of structures down to a few nanometers.

The black lines in the graphs of Fig. 9 also show that the average number of sites is near integer at $300 \mathrm{~K}$. This indicates that the ground state configuration dominates at this temperature such that the integer values are the number of step sites in the ground state configuration. At $1200 \mathrm{~K}$, the points are not so bound to the integer values, i.e., at this temperature, the higher-energy configurations are important as well. However, the graphs indicate a rather small temperature dependence on the number of sites.

Figure 10 shows the average number of these four step sites as a function of the number of atoms in the cluster in the range between 1000 and 1009 atoms. These graphs show less rapid variations in the average number of sites indicating that the single atom influence on the properties begin to loose the importance at these cluster sizes. This may be the first estimation of the size at which the properties of a cluster will cease to change dramatically due to the addition or removal of a single atom.

From the graphs in Fig. 10, it is also seen that the tendency toward having near integer values of the average number of sites at $300 \mathrm{~K}$ is not so big at these larger clusters. That is not so surprising since the number of possible configurations is much higher at larger clusters, i.e., the ground state configuration will be less dominant. Furthermore, it is seen from the graphs that a higher temperature has a stronger tendency to smear out the size effects rather than increasing the number of step sites as one might intuitively expect.

When performing real experiments, it is unfortunately impossible to measure the exact number of atoms in the clusters that are present on a sample. So in order to present some results which are more comparable to experiment, we need to 

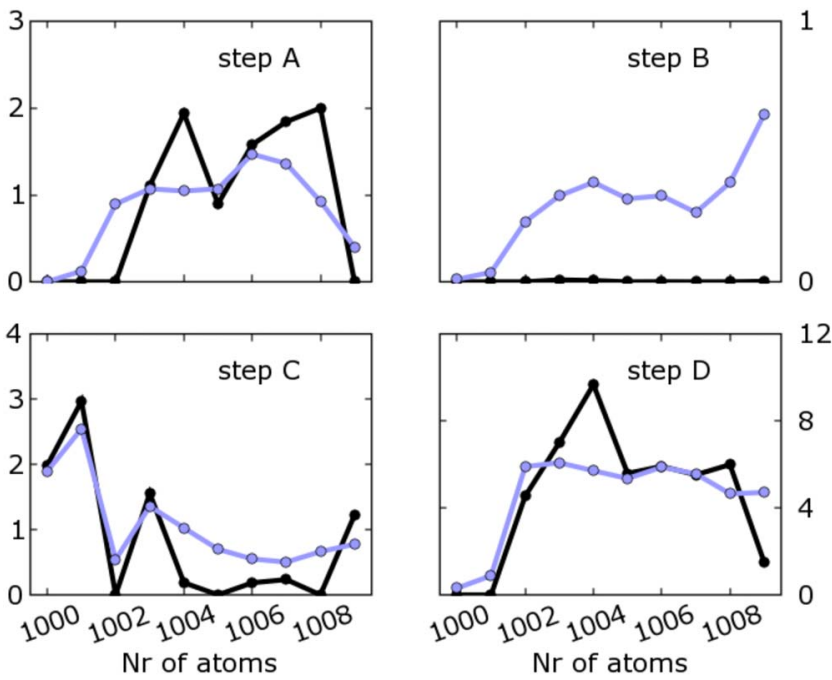

FIG. 10. (Color online) The number of step sites as a function of the number of atoms in the cluster. The four step sites are defined in Fig. 8. The black curve is at a temperature of $300 \mathrm{~K}$, whereas the gray (light blue) is at $1200 \mathrm{~K} .1000$ atoms corresponds to a cluster with approximately $2.7 \mathrm{~nm}$ diameter.

do some averaging over several cluster sizes. Figure 11 shows the number of step sites per volume at $300 \mathrm{~K}$ and at $1200 \mathrm{~K}$ as a function of cluster diameter. Each point is an average of approximately 60 different clusters evenly distributed in the diameter range of $\pm 0.1 \mathrm{~nm}$ around the desired diameter. All cluster sizes in the range contribute equally to the average, no attempt is made to take into account that some cluster sizes, for example, corresponding to closed shells, might be more abundant since this would depend on
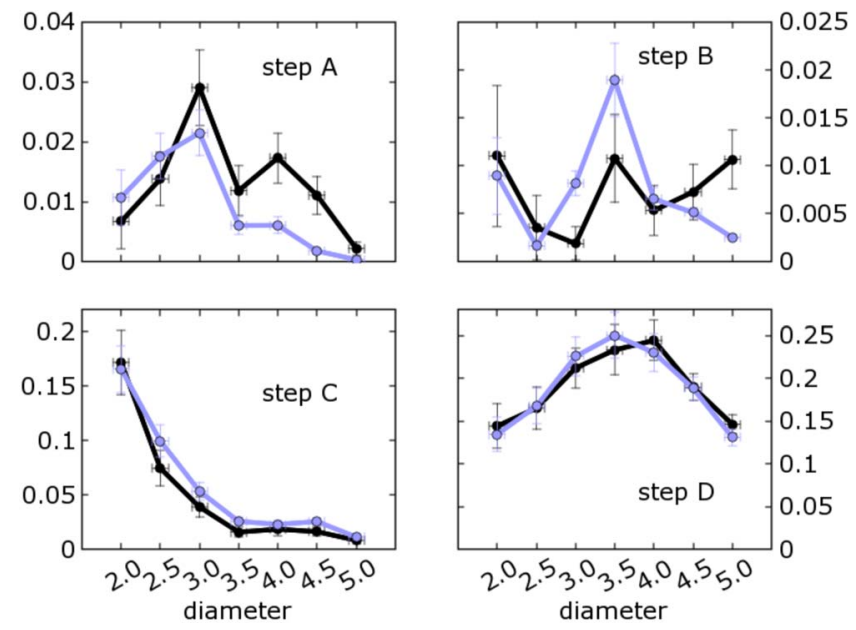

FIG. 11. (Color online) The number of step sites per volume as a function of the cluster diameter. The four step sites are defined in Fig. 8. The black curve is at a temperature of $300 \mathrm{~K}$, whereas the gray (light blue) is at $1200 \mathrm{~K}$. Each point is an average of approximately 60 different clusters. The uncertainty in the diameter indicates the spread in cluster sizes, whereas the uncertainty in the number of sites is the average standard deviation of the number of sites obtained from the 60 different clusters. The unit in the $y$ axis is $\mathrm{nm}^{-3}$. the details of how the clusters were produced.

The figures clearly indicate that the size dependency is different for the different types of sites. For step site A, there is apparently a maximum at $3 \mathrm{~nm}$, which is more significant at $1200 \mathrm{~K}$ compared to $300 \mathrm{~K}$. For step site B, there seems to be a maximum at a diameter of $3.5 \mathrm{~nm}$. However, the curve is not very smooth, especially at $300 \mathrm{~K}$, the variations are large. For step site $\mathrm{C}$, the curve seems to increase monotonically for decreasing cluster size, whereas for step site $\mathrm{D}$, the curve is smooth with a maximum around $3.5-4.0 \mathrm{~nm}$ depending on the temperature. This illustrates very well the complexity involved when trying to understand the size scaling of cluster properties. From the graphs, it is also seen that the uncertainties are smaller at $1200 \mathrm{~K}$ than at $300 \mathrm{~K}$. This is a consequence of the smearing of the cluster size effects by the temperature, which was also seen above.

The obtained values for the number of sites are quite low. It is approximately an order of magnitude lower than the rough estimates made by Honkala et al. ${ }^{14}$ For example, for step site B $\left(B_{5}\right)$, we get a peak value between 1.5 and $2.5 \mu \mathrm{mol} / \mathrm{g}$ at $3.5 \mathrm{~nm}$ in diameter where they find a peak value of $30 \mu \mathrm{mol} / \mathrm{g}$ at $2 \mathrm{~nm}$ diameter. However, our peak is a bit broader, so they would probably get approximately a factor 10 lower reaction rate, if our results for step site B was used in their work, which is a change in the wrong direction compared to experiments. However, they only include the contribution from the $B_{5}$ site and in the next section, we show that step sites A and D probably give significant contributions to the overall rate. Furthermore, it is not impossible that other factors, which are not included in the model, influence the presence of step sites. For example, it is likely that the presence of gas will increase the number of step sites since the adsorption energy typically is higher at step sites compared to flat surfaces, which would favor the presence of step sites. Furthermore, the presence of a substrate could influence the presence of step sites, at least through the limitations it would give on the topological freedom. This will be the topic of later publications.

\section{B. Activity versus cluster size}

In order to illustrate how these data on the occurrence of special sites can be used to get the catalytic activity, we have calculated the dissociation barrier of nitrogen on all four step sites since nitrogen dissociation is the rate limiting step in the ammonia synthesis. These calculations were done using density functional theory. Figure 12 shows the obtained initial, transition, and final states for all four step sites. The initial and final states have been obtained by keeping the surface atoms fixed and making a local minimization of the energy by changing the position of the nitrogen atoms. The initial states are not the lowest-energy molecular absorbed states. In all four cases, these states are found when the nitrogen molecule sticks out of the surface from the top of a surface atom. However, the energy barriers for going down to the shown initial states are much smaller than the barrier found for the actual splitting of the molecule. The barrier of dissociation is then found by making a local minimization of the energy by changing the position of the nitrogen molecule 

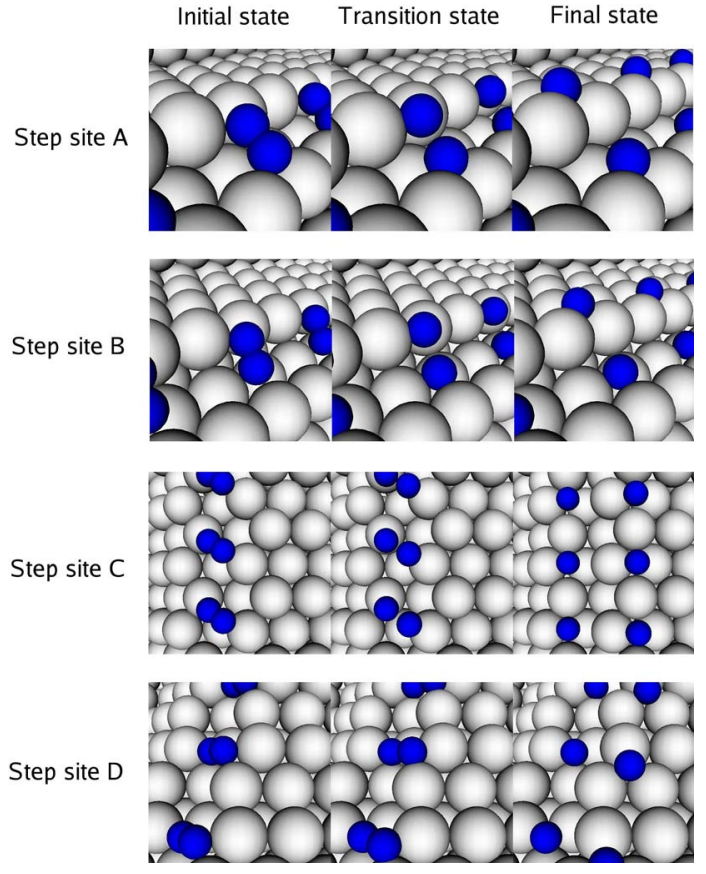

FIG. 12. (Color online) The splitting of a nitrogen molecule on the four different step sites defined in Fig. 8. The initial states are just local minima and not the lowest-energy absorption states. However, in all cases, the barrier between the lowest-energy absorption states and the initial state is lower than the barrier between the initial state and the final state, i.e., the energies of the transition states are the true barrier energies for the dissociation.

for different fixed values of the molecular bond length. From that, we get the energy as a function of bond length, which is shown on Fig. 13 for all the four different step sites.

All barrier heights are compared to the gas phase level since this is the relevant activation energy at relevant pressures and temperatures, where the coverage is low. ${ }^{10}$ From

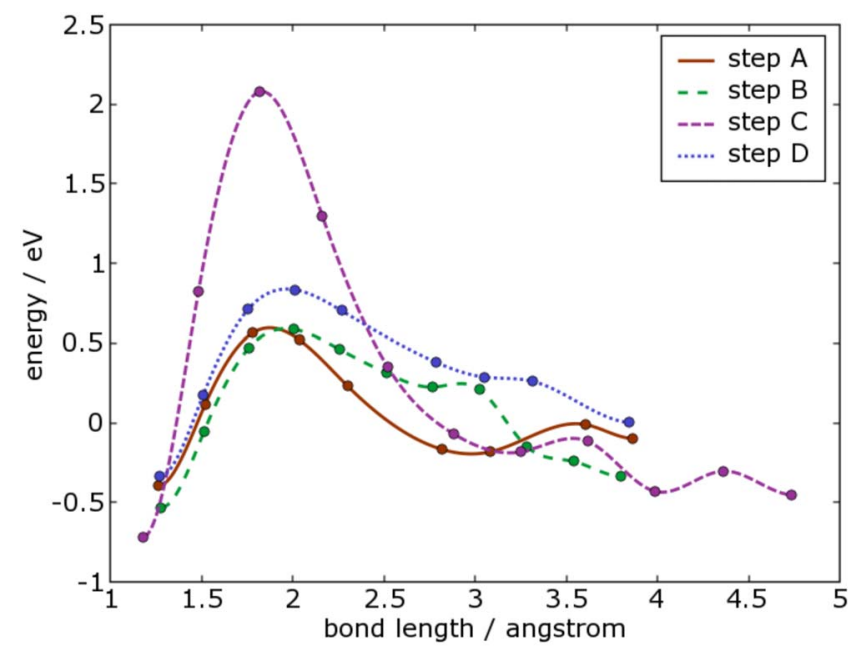

FIG. 13. (Color online) The energy as a function of the nitrogen bond length for the dissociation of a nitrogen molecule on the four different sites defined in Fig. 8. All energies are relative to the gas phase energy. The lines are cubic splines connecting the actual calculation points. this, we see that step site $\mathrm{C}$ has a large barrier of more than $2 \mathrm{eV}$ with respect to the gas phase level, which means that it is practically catalytic inactive. The barrier height is even higher than the barrier on the flat close-packed surface (0001). ${ }^{25}$ The barrier is approximately the same for step sites $\mathrm{A}$ and $\mathrm{B}$, i.e., $0.59 \mathrm{eV}$, with respect to gas phase level. This is a bit higher than what has earlier been reported for step site $\mathrm{B}$ (Ref. 10), which is known as the $B_{5}$ site. This difference is due to the fact that we calculate the barrier for a step sitting on the edge of a cluster, whereas Ref. 10 calculates the barrier for a step sitting on a surface, i.e., the neighboring environment is a bit different. The energy barriers on the nonstepped edges in Fig. 8 are much higher, so they do not contribute to the activity.

A very simple approximation of the catalytic activity in the ammonia synthesis for the four different step sites can be obtained from the found barrier heights by writing the activity as

$$
r=A \exp \left[-\frac{E_{a}}{k_{B} T}\right]
$$

where $E_{a}$ is the barrier height with respect to gas phase level. We do not include the adsorbed molecular state explicitly since it is in equilibrium with the gas phase and has a low coverage at relevant pressures and temperatures. ${ }^{10} k_{B}$ and $T$ are the Boltzmann constant and the temperature, respectively. $A$ is the prefactor, which depends on the equilibrium constants of the other reaction steps and the gas pressures. However, we assume that the prefactor is the same on all the sites since we only need a rough estimate of the activity of the different cluster sizes relative to each other. In this way, we avoid making detailed calculations of all the elementary partial reactions in the ammonia synthesis for all the differ-

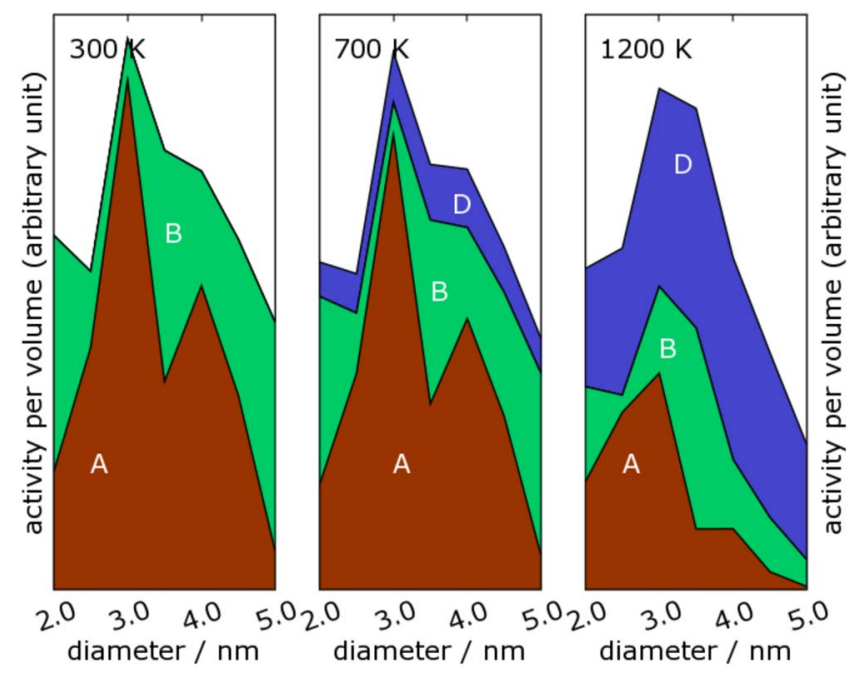

FIG. 14. (Color online) The total catalytic activity of ruthenium clusters in the ammonia synthesis as a function of the cluster diameter. The different colors indicate how much the different step sites contribute to the total activity. The activity peaks at a cluster size of $3 \mathrm{~nm}$. 
ent sites, as it was done for the $B_{5}$ site by Logadóttir and Nørskov. ${ }^{10}$

The catalytic activities obtained from Eq. (2) can then be multiplied by the number of sites present on the clusters to obtain the catalytic activity per volume catalyst as a function of cluster diameter, which is seen in Fig. 14. The graphs at 300 and $700 \mathrm{~K}$ indicate a maximum in the catalytic activity per volume catalyst at a cluster diameter of $3 \mathrm{~nm}$, even though the curve is not so smooth. At $300 \mathrm{~K}$, it is clearly seen that all the catalytic activity comes from step sites A and $\mathrm{B}$, whereas step site D start contributing at $700 \mathrm{~K}$. At $1200 \mathrm{~K}$, the picture is a bit more clear and smooth. There still seems to be a optimal cluster diameter at $3 \mathrm{~nm}$. The existence of such a maximum fits well with the experimental observation that the catalytic activity of a sample can increase after sintering the smallest Ru particles. ${ }^{26}$ Furthermore, it is seen that step site D also contributes to the total activity at this higher temperature due to fact that it is more present on the clusters than the two others and the fact that the difference in barrier height becomes less important as temperature increases. The ammonia synthesis typically runs at a temperature around $700 \mathrm{~K}$ in industrial catalysts.

\section{SUMMARY}

We have developed a method to determine detailed structural information of ruthenium clusters in the diameter range from 1.5 to $5 \mathrm{~nm}$. The method can directly be applied to other hcp metals and with minor modifications, it can be applied to other structures as well. We find that the number of catalytic active step sites does not change much with temperature within reasonable temperature ranges. The role of the temperature is more to smooth out some of the strong size dependence. At very small cluster sizes, the structure depends sensitively on the exact number of atoms. We find that this strong single atom dependence begin to cease at cluster sizes around 1000 atoms. We find that the dependence on the presence of step sites with respect to the cluster diameter is significantly different for the different types of step sites. This illustrates the complexity involved in understanding size dependencies. Finally, we made density functional theory calculations in order to get the barrier of dissociation for nitrogen on the different step sites. This barrier was used to estimate the ammonia synthesis activity of the different step sites. This information was combined with the structural information to obtain the catalytic activity versus cluster size. Here, we found that the optimal cluster diameter is approximately $3 \mathrm{~nm}$.

\section{ACKNOWLEDGMENTS}

The authors wish to thank J. K. Nørskov and I. Chorkendorff for fruitful discussions and for reading this paper. The Center for Individual Nanoparticle Functionality (CINF) is sponsored by the Danish National Research Foundation. This work was supported by the Danish Center for Scientific Computing. *schiotz@fysik.dtu.dk

${ }^{1}$ E. H. G. Backus, A. Eichler, M. L. Grecea, A. W. Kleyn, and M. Bonn, J. Chem. Phys. 121, 7946 (2004).

${ }^{2}$ M. A. van Daelen, M. Neurock, and R. A. van Santen, Surf. Sci. 417, 247 (1998)

${ }^{3}$ N. M. Galea, D. Knapp, and T. Ziegler, J. Catal. 247, 20 (2007).

${ }^{4}$ B. Hammer, Surf. Sci. 459, 323 (2000).

${ }^{5}$ S. Kandoi, J. Greeley, M. A. Sanchez-Castillo, S. T. Evans, A. A. Gokhale, J. A. Dumesic, and M. Mavrikakis, Top. Catal. 37, 17 (2006).

${ }^{6}$ I. N. Remediakis, F. Abild-Pedersen, and J. K. Nørskov, J. Phys. Chem. B 108, 14535 (2004).

${ }^{7}$ M. Mavrikakis, J. Rempel, J. Greeley, L. B. Hansen, and J. K. Nørskov, J. Chem. Phys. 117, 6737 (2002).

${ }^{8}$ J. G. Wang and B. Hammer, J. Chem. Phys. 124, 184704 (2006).

${ }^{9}$ Y. Zhu, Y. Dai, D. Chen, and W. Yuan, J. Mol. Catal. A: Chem. 264, 299 (2007).

${ }^{10}$ Á. Logadóttir and J. K. Nørskov, J. Catal. 220, 273 (2003).

${ }^{11}$ S. Linic and M. A. Barteau, J. Am. Chem. Soc. 125, 4034 (2003).

${ }^{12}$ K. Reuter and M. Scheffler, Phys. Rev. B 73, 045433 (2006).

${ }^{13}$ S. Dahl, Á. Logadóttir, R. C. Egeberg, J. H. Larsen, I. Chorkendorff, E. Törnqvist, and J. K. Nørskov, Phys. Rev. Lett. 83, 1814 (1999).

${ }^{14}$ K. Honkala, A. Hellman, I. N. Remediakis, A. Logadottir, A. Carlsson, S. Dahl, C. H. Christensen, and J. K. Nørskov, Science 307, 555 (2005).
${ }^{15}$ K. W. Jacobsen, P. Stoltze, and J. K. Nørskov, Surf. Sci. 366, 394 (1996).

${ }^{16}$ K. W. Jacobsen, J. K. Nørskov, and M. J. Puska, Phys. Rev. B 35, 7423 (1987).

${ }^{17}$ B. Hammer, L. B. Hansen, and J. K. Nørskov, Phys. Rev. B 59, 7413 (1999).

${ }^{18}$ D. Vanderbilt, Phys. Rev. B 41, 7892 (1990).

${ }^{19}$ G. Kresse and J. Furthmuller, Comput. Mater. Sci. 6, 15 (1996).

${ }^{20}$ The DACAPO code is available as a part of the CAMPOS software (www.camp.dtu.dk/Software).

${ }^{21} \mathrm{We}$ use the form of the EMT potential which is described by Jacobsen et al. (Ref. 15). The potential was optimized to give most accurate cohesive energy, lattice parameters, elastic constants, and surface energies possible for Ruthenium by changing six parameters defined by Jacobsen et al. (Ref. 15). We found these optimal values: $E_{0}=-6.3505 \mathrm{eV}, V_{0}=12.3052 \mathrm{eV}, s_{0}$ $=1.4984 \AA, \quad \eta_{2}=3.9452 \AA^{-1}, \quad \kappa=6.5612 \AA^{-1}, \quad$ and $\lambda$ $=6.7015 \AA^{-1}$.

${ }^{22}$ T. W. Hansen, J. B. Wagner, P. L. Hansen, S. Dahl, H. Topsøe, and C. J. H. Jacobsen, Science 294, 1508 (2001).

${ }^{23}$ B. Hammer and J. K. Nørskov, Nature (London) 376, 238 (1995).

${ }^{24}$ B. Hammer and J. K. Nørskov, Surf. Sci. 343, 211 (1995).

${ }^{25}$ J. J. Mortensen, Y. Morikawa, B. Hammer, and J. K. Nørskov, J. Catal. 169, 85 (1997).

${ }^{26}$ C. J. H. Jacobsen, S. Dahl, P. L. Hansen, E. Törnqvist, L. Jensen, H. Topsøe, D. V. Prip, P. B. Møenshaug, and I. Chorkendorff, J. Mol. Catal. A: Chem. 163, 19 (2000). 